\title{
Fundamentos epistemológicos de la clasificación documental
}

\author{
Miguel Angel Esteban Navarro \\ Area de Biblioteconomía y Documentación \\ Universidad de Zaragoza
}

«We may conclude that in the fundamental sense: There is no substitute for classification». (Derek W. Langridge, Classification, 1992).

\subsection{Resumen}

La elaboración de una teoría de la Organización del Conocimiento capaz de explicar y afrontar los retos actuales, exige la construcción de un concepto preciso de sus elementos básicos desde una perspectiva interdisciplinar. Así, se presentan los rasgos más relevantes de los procesos documentales de ordenación, clasificación y organización a partir del análisis de algunos de los caracteres de los recursos cognitivos que el hombre pone en acción para construir conceptos y comprender el mundo, como la capacidad de ordenar, clasificar y organizar. Se destaca también como un elemento positivo para el tratamiento documental, el hecho de que la actividad clasificatoria se efectue siempre mediante criterios selectivos y relacionales. A continuación, se critican las aproximaciones descriptivistas y reduccionistas a la clasificación documental, para proponer un concepto que parta de la observación del proceso intelectual analítico y sintético que se pone en acción durante su ejercicio, con independencia del centro de información en el que se efectue y el lenguaje documental que se utilice. Se termina con un ensayo de definición que atiende a la dimensión operativa e instrumental de la clasificación documental, presentando los ejes que deben articular una elaboración más profunda. (Autor)

Palabras clave: Teoría de la Clasificación. Epistemología. Proceso documental.

\subsection{Abstract}

Building an explicative and pragmatic theory of the Knowledge Organization requires the elaboration of a precise conceptualization of its basic elements from an interdisciplinary perspective. Therefore, the analysis of three cognitive human

Scire. 1 : 1 (en.-jun. 1995). 
resources to build concepts and to understand the world (the capacity of order, classify and organize), serves to explain the most characteristic qualities of the documentary processes of ordering, classifying and organizing. Classifying by means of the selective and relational criteria is considered positive for the information retrieval. The descriptive and reductionist conceptions of the documental classification are discussed. Alternatively, a concept based on the observation of the analytical and synthetical intellectual process of the indexing and classifying is propossed. So, a unique definition is essayed for all kind of information centers and documentary languages. (Author)

Keywords: Classification Theory. Episthemology. Information Chain.

\section{Introducción}

La construcción de una teoría explicativa de los fundamentos, elementos, estructura y funciones de los procedimientos y herramientas utilizados para representar el contenido de los documentos, que garantice el diseño de un eficaz sistema de conservación, recuperación y comunicación de los conocimientos que éstos albergan, es uno de los principales retos que la Organización del Conocimiento ha de afrontar en su intento de consolidarse como una disciplina científica. El norte de esa reflexión teórica debe ser la investigación aplicada a la mejora de la gestión de las técnicas del proceso documental y, en particular, de los lenguajes documentales.

Por fortuna, hasta el presente, los avances en la práctica de la representación, organización y comunicación documental del conocimiento han sido ilustrados, e incluso dirigidos en algunas ocasiones, por una labor de investigación básica. A su vez, esta investigación siempre ha asumido como principal objetivo proporcionar a los gestores de la información unos criterios de actuación y unos recursos instrumentales que, por sustentarse sobre una base científica, permitan solventar los problemas que surgen durante el ejercicio de su trabajo e idear nuevas tareas y herramientas para llevarlas a cabo. De este modo, varios modelos teóricos se han sucedido desde finales del siglo XIX en un proceso, en general, acumulativo de conocimientos pero tampoco exento de rupturas. Sin embargo, en la actualidad, nos encontramos en un momento crítico de reformulación de las hipótesis y los contenidos básicos de la Organización del Conocimiento, gracias, entre otros fenómenos, al desarrollo de un paradigma informacional en las ciencias, a la formación de un nuevo terreno de reflexión interdisciplinar denominado Ciencias Cognitivas y a la aparición de las nuevas tecnologías de la información. De ahí, la conveniencia de revisar algunos de sus principios y de las respuestas dadas a los interrogantes del pasado, así como de plantear nuevos problemas que nos permitan seguir avanzando sobre una base firme en la orientación expuesta.

Scire. 1 : 1 (en.-jun. 1995). 
Entre los contenidos mínimos de cualquier teoría que aspire a aglutinar los múltiples elementos y perspectivas de acercamiento a los problemas presentes en un panorama de abierta recomposición, consiguiendo una explicación operativa en la praxis, deben figurar al menos los siguientes, en una lista que no pretende ser exhaustiva. La caracterización del sistema cognitivo y comunicacional humano, complementada con una exposición de las aportaciones efectuadas por las disciplinas dedicadas a su estudio, que más útiles sean desde una perspectiva documentaria. La reflexión sobre la evolución de las ideas y los instrumentos de relación, representación, ordenación, clasificación y organización en el pensamiento occidental durante los dos últimos siglos. La exposición de una historia de la clasificación e indización desde una perspectiva que, en lugar de efectuar una mera enumeración y descripción de los diversos sistemas, se dedique a comprender su significado en el marco de su tiempo y en el de la historia global de la Documentación, con objeto de explicar la situación presente y descubrir sus posibles aportaciones para modelar su evolución. La presentación de los fundamentos lingüísticos y semióticos de la comunicación humana y de las técnicas documentales, incidiendo en las posibilidades y límites que el análisis de contenido encuentra derivados del carácter del lenguaje humano. El diseño de una metodología que establezca los principios básicos para la definición de los caracteres propios de un sistema de tratamiento y recuperación de la información y de sus criterios de construcción y evaluación. Y el análisis del impacto que la revolución informática está teniendo en la mecanización de los procesos documentales, en el diseño de herramientas inteligentes de representación y organización del conocimiento y en los sistemas y estrategias humanas de acceso, comunicación y difusión de la información.

En las dos colaboraciones que presentamos en esta publicación nos limitamos a bosquejar únicamente algunos elementos de esas tres primeras materias de estudio, ya que por encontrarnos todavía inmersos en su diseño, sólo podemos plantear interrogantes y desvelar conexiones, pero sin que eso suponga renunciar a proponer vías de solución a los problemas planteados y a avanzar las primeras conclusiones sobre algunos caracteres de los fundamentos epistémicos de la acción de organizar. En concreto, en este primer texto, analizamos los caracteres más relevantes de algunos de los recursos conceptuales que el documentalista pone en acción durante su trabajo, como la capacidad de ordenar, clasificar y organizar. Para sobre esta base, y a partir de la exposición de los rasgos más relevantes de los procesos de indización y clasificación, presentar los componentes fundamentales de un concepto integrado de clasificación documental. Reservando, para la siguiente colaboración, la defensa de cómo se enriquece el estudio de un terreno bastante descuidado: la historia del concepto y de la tipología de los lenguajes documentales, si se realiza con una orientación interdiscipli-

Scire. 1 : 1 (en.-jun. 1995). 
nar que muestre la estrecha ligazón de su evolución con respecto a algunos de los problemas epistemológicos más relevantes de nuestra cultura, como la incesante búsqueda de un método eficaz de preservación y transmisión del conocimiento humano para generar nuevo conocimiento.

Con estos trabajos pretendemos colaborar en la resolución de una tarea básica e ineludible para llevar la empresa expuesta más arriba a buen puerto: elaborar un concepto preciso de "organización del conocimiento", ya que únicamente la previa definición y análisis de qué es el conocimiento y de qué se entiende por representar, organizar y comunicar, nos puede ayudar tanto a establecer los fundamentos epistemológicos y cognitivos del objeto y las acciones de esta nueva ciencia, como a reflexionar sobre las posibilidades y límites de las prestaciones de los lenguajes documentales. Lo cual, a su vez, nos dará la base sobre la que edificar un concepto y una teoría de la clasificación documental dentro del marco común de la Organización del Conocimiento, desde una perspectiva integrada e integradora que diriga la investigación por dos vías. Por un lado, hacia sí misma y su propio ámbito práctico de acción, acabando con la confusión de teoría de la clasificación con teoría de la clasificación bibliográfica, mediante la integración de esta última con los estudios sobre los fundamentos de los lenguajes de indización y de los sistemas de clasificación y descripción archivísticos. Y por otro lado, hacia el exterior, analizando todas las implicaciones que se tejen en torno a los procesos humanos de representar, organizar y comunicar el conocimiento y el diseño de herramientas lógicas y materiales que faciliten su desenvolvimiento durante el trabajo documental.

\section{Ordenar, clasificar y organizar}

El conocimiento es una capacidad consustancial de la naturaleza del hombre y un requisito indispensable para garantizar su supervivencia en el mundo y su desarrollo como ser físico y moral. Precisamente, la aparición de un modo singular de conocer la realidad, caracterizado por permitir una comprensión del entorno capaz de guiar una conducta adaptativa y transformadora, generar una cultura y expresar las ideas y los sentimientos mediante un lenguaje articulado y simbólico, permitió en el pasado a unos primates adquirir la condición de homínidos. Desde su mismo origen, la formación del conocimiento humano se halla ligada con la evolución histórica de las diversas concepciones del mundo, de los hábitos y comportamientos sociales basados en esas imágenes y de los sistemas de comunicación naturales y artificiales que controlan e inspiran, en íntima correlación entre sí, las interacciones materiales, energéticas e informativas de los individuos y de las sociedades humanas con el medio ambiente y el resto de sus congéneres. Conocimiento, pensamiento creativo, cultura y lenguaje se conjugan formando las cuatro dimensiones esenciales del ser humano: homo sapiens, homo ludens,

Scire. $1: 1$ (en.-jun. 1995). 
homo faber y homo loquens, que lo singularizan frente al resto de los animales superiores.

Asimismo, el conocimiento humano surge de la relación del hombre con su entorno físico y social, ya que se trata, básicamente, del resultado de organizar conceptualmente la percepción y representación de las entradas de información bajo las que se presentan, o a las que son reducibles, sus interacciones, tras su recepción por un sofisticado sistema de información. Es decir, la comprensión de la realidad se efectua mediante la recepción por los sentidos de señales producidas por nuestra acción y experiencia, que, a continuación, son formalizados y convertidos en conceptos, que tras su organización e interpretación dan lugar al conocimiento. Esa percepción del mundo es el resultado del estado en el que se encuentran como mínimo dos factores: por un lado, el mundo exterior; y, por otro, nuestro aparato sensorial, convenientemente auxiliado por instrumentos artificiales cada vez más precisos y eficientes en su tarea. Pero la representación y comunicación inteligente de lo percibido —que permite nuestra integración y acción en la realidad - también depende, fundamentalmente, de los caracteres que adoptan otros dos factores estrechamente ligados entre sí: nuestro sistema conceptual y nuestro sistema lingüístico. La suma de esos cuatro factores modela toda la estructura cognitiva y comunicacional humana, correspondiendo, principalmente, al sistema conceptual humano seleccionar, condicionar y determinar los aspectos del mundo que tenemos en cuenta, en los que pensamos y de los que hablamos.

En definitiva, una buena parte del conocimiento comienza allí donde cesa la observación, para pasar a tareas intelectuales más complejas. Porque el conocimiento humano no consiste simplemente en recopilar datos para acumularlos en montones sin orden ni concierto, sino que en la medida en que es un conocimiento racional y crítico, se trata ante todo de un intento de organizar y representar los hechos observados de una forma coherente y sistemática dentro de la estructura articulada de un lenguaje, si bien condicionado por el marco sociocultural en el que se desarrolla y aplica. Posteriormente, el conocimiento se convierte de nuevo en información cuando es transmitido por los hombres bajo la forma de actos y mensajes, que serán de nuevo transformados en conocimiento tras su recepción y procesamiento por otros hombres; cerrándose, de este modo, el ciclo comunicacional del conocimiento humano.

Esta concepción del conocimiento humano como un proceso al mismo tiempo cognitivo, informacional y comunicacional, nos permite establecer como pilares de su construcción no sólo los procesos sensitivos y mentales de percepción, representación y organización, sino también, con el mismo grado de importancia, los procesos comunicativos y las acciones sociales en las que se manifiesta. El estudio de los procesos comunicativos humanos desde una perspectiva docu-

Scire. $1: 1$ (en.-jun. 1995). 
mentaria exige una exposición general de los conceptos de información y comunicación, un análisis del tratamiento y la recuperación de la información como interacción comunicativa y una reconsideración de la comunicación documental como un acto, ante todo, de transmisión lingüística del conocimiento, para fundar sobre sus conclusiones una estrategias de optimización de la representación documental del conocimiento. No obstante, aquí sólo nos ocuparemos del análisis de algunos de los elementos del proceso mental de organización.

Con facilidad observamos cómo el hombre hace uso continuamente y de modo inconsciente de recursos organizativos, que le sirven para facilitar su vida diaria y actuar con eficacia en el mundo. Dentro de esa actividad organizativa inconsciente se distinguen dos ámbitos de acción: la ordenación y clasificación de objetos para facilitar su gestión (ordenar la ropa en un armario, disponer la vajilla para su limpieza, etc.) y la ordenación y clasificación de ideas para facilitar la comprensión de la realidad (por ejemplo, la decisión de que pensamientos son buenos, malos, indiferentes, perdonables, injustificables... desde unos principios morales aceptados irreflexivamente). El hombre su auxilia para efectuar tales tareas de los conceptos, que expresa mediante las palabras, que, desde esta perspectiva, pueden ser consideradas como los más primitivos recursos de clasificación, ya que muchas de ellas denominan clases. Los nombres propios sirven para indicar entidades únicas; los nombres comunes, excepto los nombres concretos, denotan clases amplias; y los adjetivos, combinados con los nombres, reducen o amplian el campo de referencia de las clases (Langridge, 1992, p. 3-4).

Aunque estos actos mentales y lingüísticos parecen, aparentemente, bastante simples, de hecho no lo son, pues forman parte de un complejo proceso desarrollado por el sistema humano para construir su conocimiento de la realidad. Simplificando, comprender la naturaleza implica recoger experiencias a través de los sentidos, que más tarde son formalizados y convertidos en conceptos y discursos por la razón mediante un proceso que pone en acción, fundamentalmente, cuatro recursos del sistema cognitivo: dividir, ordenar, clasificar y organizar información para generar conocimiento, cuyos resultados expresa mediante palabras y acciones.

La división consiste en distribuir los elementos de un universo en una serie de grupos exhaustivos y disyuntos dos a dos —es decir, que juntos cubran toda su extensión y que no mantengan ningún miembro en común-, sin que exista ninguna relación sistemática entre ellas. La división más simple consiste en la dicotomía, basada en el uso de un sólo concepto: así, el concepto de susceptible de ser comido, sugiere la divisón de todos los seres vivos en comestibles y no comestibles.

Después de la división, sigue en orden de complejidad la ordenación de esa

Scire. 1 : 1 (en.-jun. 1995). 
realidad, mediante el establecimiento de una relación asimétrica y transitiva entre sus elementos, basada en la elección de un único criterio relacional. Es decir, por ordenar se entiende situar y conectar los elementos en un contexto espacio-temporal o en otras dimensiones de acuerdo a un solo criterio. Por ejemplo, la ordenación de los elementos químicos según su número atómico.

A continuación, actúa la clasificación, que puede ser definida como «el modo más simple de discriminar simultáneamente los elementos de un conjunto y de agruparlos en subconjuntos, o sea, el modo más simple de analizar y sintetizar» (Bunge, 1983, p. 95). En un sentido amplio, clasificar significa «juntar cosas o ideas semejantes, y separar las que son diferentes» (Vickery, 1975, p. 1); es decir, se trata de la «acción de agrupar objetos en clases según las propiedades comunes descubiertas o los resultados de tales agrupaciones» (Runes, 1985, p. 60). De modo más preciso, por clasificar se entiende la sucesión de tres procesos por este orden: la distinción de elementos dentro de la complejidad de lo real mediante un único e irrepetible criterio; su agrupación en clases por similitudes o dimensiones relevantes de sus caracteres, que intentan esclarecer y reconstruir relaciones existentes en la naturaleza del universo que forman los elementos; y la construcción de criterios de comparación. Resultado de la clasificación es la distinción entre géneros y especies; de modo que en el lenguaje filosófico también se entiende por clasificar la «acción de agrupar especies en géneros, y géneros en géneros más amplios hasta llegar al summum genus (el género que no es especie de otro género superior)» (Runes, 1985, p. 60).

Y por organizar se entiende el resultado de seleccionar, depurar, almacenar y desechar los criterios de ordenación y clasificación de los elementos, y de construir y controlar las relaciones entre las clases, de acuerdo con diversos parámetros, para optimizar la acción y la comprensión de otros fenómenos; construyendo mediante estas acciones un sistema de conocimiento progresivamente más y más complejo.

\section{Selección y relación en el proceso cognitivo humano}

Las tareas de identificación y de organización constituyen los requisitos básicos del proceso de descripción y comprensión de los fenómenos que se producen en la realidad. La identificación nos permite discernir los casos específicos unos de otros. En cambio, la organización nos descubre que esos entes y hechos pertenecen a cierto tipo determinado, que comparten propiedades con otros ejemplares de una misma clase y que dentro de las clases y entre ellas se producen una serie de relaciones, cuya exposición nos acerca a la plena comprensión del mundo. Y esto último es realmente lo más importante, porque el conocimiento humano, y en particular la indagación científica, nunca se propone, únicamente, señalar o identificar casos o ejemplos singulares, sino descubrir las relaciones o

Scire. 1 : 1 (en.-jun. 1995). 
pautas que siguen los hechos, para organizarlos o ligarlos entre sí de alguna manera inteligible. Por tanto, la organización consiste en cooperar en la tarea de representar los hechos y los objetos, de forma que no nos limitemos a identificaciones particulares y aisladas unas de otras, sino favoreciendo (permitiendo) su identificación y comprensión en un contexto, en un marco de relaciones con otros hechos. Selección y relación forman la clave de la bóveda del proceso de representación y organización de la realidad.

El hombre procede a organizar los fenómenos, los objetos y las ideas de acuerdo con una selección de sus rasgos que reciben el nombre de propiedades, atributos o cualidades — - según la convención que utilicemos-, persiguiendo el hallazgo y fijación de los nexos y relaciones que los unen en su desenvolvimiento. Pero esa selección se efectua con una perspectiva condicionada por el entorno en el que se produce y los objetivos que se pretenden conseguir. De ahí que el proceso de organización de una misma realidad por distintos individuos suele generar diversos resultados.

Ciertamente, un objeto o una idea pueden clasificarse de muy diversas formas. Asimismo, una unidad clasificatoria se asigna a una clase de acuerdo con la posesión de unas características comunes por parte de los elementos de esa misma clase, pero esa unidad puede remitir a otra clase si varía el interés clasificatorio predominante o la perspectiva que adoptamos. Porque cuando representamos y organizamos en nuestro pensamiento la realidad, elegimos (seleccionamos) ciertos atributos o propiedades de los objetos para distinguirlos entre sí, al tiempo que despreciamos o pasamos por alto otros, en función del interés de nuestra clasificación. Es decir, nos enfrentamos con la realidad de un modo selectivo: delimitamos rigurosamente qué aspectos consideramos pertinentes o en qué propiedades de las cosas fijamos nuestra atención, dejando de lado todo lo demás. Por ejemplo, al clasificar las características físicas a registrar en el estudio de una población, podemos emplear parámetros tales como la estatura, el peso, la edad y el tipo sanguíneo, omitiendo totalmente las opiniones políticas, el color del pelo y el nombre de las personas sometidas a la encuesta, a las que identificaremos valiéndonos simplemente de un número respetuoso del anonimato. De todo lo cual resulta tanto una amplia posibilidad de representar y organizar los elementos de un universo según un extenso abanico de posibilidades, como una enorme variedad y un alto riesgo de variabilidad de los sistemas de organización.

Esta variedad y variabilidad de los sistemas de clasificación y de ordenación se debe al hecho de que estos sistemas no son algo concreto que se encuentran en la naturaleza, no son unos fenómenos que el hombre debe descubir, sino a que son el resultado de un recurso de la naturaleza humana que el hombre usa para enfrentarse y comprender la realidad. Y, por tanto, como resultado del uso de esa capacidad, la clasificación consiste en una construcción artificial y una imagen

Scire. 1 : 1 (en.-jun. 1995). 
ordenada de la naturaleza realizada por cada hombre en función de sus intereses y necesidades, a partir de la selección de unos rasgos que él considera relevantes, de entre las innumerables posibilidades clasificatorias o criterios selectivos que le ofrece la naturaleza con su enorme diversidad de elementos. En sentido más estricto, la representación de la realidad exige la elección de ciertos parámetros selectivos.

No obstante, la diversidad clasificatoria está restringida por la tendencia del hombre a guiarse durante la agrupación de los elementos en clases por tres tipos de criterios o parámetros de clasificación dominantes. El criterio de cantidad: el número de rasgos en común entre los elementos de una clase. El criterio de calidad: la relevancia estructural de algún rasgo. Y el criterio de funcionalidad: elementos pragmáticos condicionados por la tarea en la que se inserta la clasificación y su utilidad o servicio. Pensemos en la clasificación de los animales en un ecosistema, el criterio de cantidad estaría representado por la elección como elementos clasificatorios del color, el tamaño y el modo de alimentación; el de calidad por el esqueleto; y el de funcionalidad, la distinción entre parásitos y beneficiosos desde una perspectiva agrícola.

En definitiva, no existe un sistema de clasificación esencial, natural o único que sea adecuado a priori a la naturaleza de la realidad. En consecuencia, no ha lugar juzgar en abstracto si una clasificación es mejor que otra, sino que para ello habrá que atender el contexto en el que se ha construido y al propósito que persigue. Como máximo, podemos mantener que un sistema clasificatorio es superior a otro porque puede aplicarse en un número mayor de contextos, porque desvela un mayor número de relaciones entre los hechos clasificados y porque posee una estructura más rigurosa.

Hemos observado como el hombre representa la realidad seleccionando atributos mediante una abstracción y discriminación de semejanzas y diferencias, es decir, a partir de lo que acerca y separa, une y distancia, a las cosas y los fenómenos. Sin embargo, organizar la realidad no es únicamente hacer fotografías aisladas de los hechos y de las cosas, rotular individuos únicos e independientes unos de otros, sino que es, ante todo, descubir y establecer relaciones. Por consiguiente, una clasificación debe proponer itinerarios, sugerir caminos entre los árboles del esquema taxonómico, y, por tanto, entre los documentos, cuando se efectua en un sistema documental. Porque si conocer consiste en representar los hechos, en someterlos a un proceso de abstracción construyendo con ellos un edificio conceptual que reproduzca la realidad, surge como una tarea fundamental hacer explícitas las relaciones existentes entre los hechos, así como expresar la forma que adoptan tales relaciones.

En este sentido, los nombres que ponemos a las cosas remiten siempre a sis-

Scire. 1 : 1 (en.-jun. 1995). 
temas de clasificación en los que esos nombres se conectan entre sí, de acuerdo en buena medida a los enlaces que se producen en la realidad. Por ejemplo, la placa de matrícula de un vehículo no se limita a señalar unívocamente ese automóvil, sino que lo identifica también como miembro de una clase de coches, a saber, la de los matrículados en una cierta provincia y nación. Asimismo, el nombre impuesto a una persona se une al apellido, con lo que se marca a tal individuo como miembro de un determinada familia. Tales clasificaciones se llevan a cabo desvelando y apoyándose en ciertas propiedades o atributos compartidos por todos los miembros de una clase dada. Así, el apellido indica que todos los miembros de la familia a la que remite tienen unos antepasados comunes, y la placa de la matrícula, que todos los automóviles que la llevan han quedado registrados en la jefatura de tráfico de una determinada provincia. Por consiguiente, la agrupación de los ciudadanos de un país por apellidos y dentro de cada apellido por familias origina una clasificación; en cambio, su presentación en una lista alfabética sólo consiste en una ordenación.

\section{Manifestaciones del proceso cognitivo humano en la organización documental}

Esas diferencias entre las acciones de ordenar, clasificar y organizar nos permiten conceptualizar sobre unos cimientos sólidos los procesos documentales denominados con esos mismos apelativos, ya que si éstos consisten en la aplicación de esos recursos del proceso cognitivo humano a un entorno particular y para desarrollar una actividad determinada, deberán asumir los caracteres generales de los que les sirven de base. Así, por organización documental entendemos el establecimiento de relaciones de dependencia entre los documentos de un fondo según unas reglas predefinidas que afectan al número y armonía de las relaciones, medidas en términos de unidades de información; las cuales son el resultado de los procesos de identificación, ordenación y clasificación de su contenido a partir de unos elementos significativos, condicionando, por consiguiente, las posibilidades y las estrategias diseñadas para su recuperación. La clasificación consiste en dividir y agrupar, según unos principios y reglas prefijados, los documentos en clases; las cuales adquieren pleno sentido mediante su integración en una jerarquía construida a partir de la elección de unas categorías y unos criterios de estructuración, que se encuentra condicionada, a su vez, por la previa asunción de unas determinadas imágenes del sistema de clasificación ideal y de la parcela del conocimiento sobre la que opera la acción de clasificar. Entendiendo por ordenación, la acción de proceder dentro de cada clase, grupos de clases o de modo global, a la disposición de los documentos en una sucesión siguiendo un criterio de relación, predefinido, único y uniforme (alfabético, numérico o alfanumérico); que puede encontrar su correlato en la instalación física en el depósi-

Scire. $1: 1$ (en.-jun. 1995). 
to documental. La ordenación y la clasificación tienen en común el hecho de que comparan los elementos de un conjunto; sin embargo, se diferencian en que la primera es lineal y unidimensional mientras que la clasificación es polidimensional, ya que como mínimo posee dos niveles de relación.

Los caracteres que adopta el proceso natural de representación y organización de la realidad también aparecen durante la construcción y la regulación del funcionamiento de los sistemas artificiales de organización del conocimiento, ya que éstos sólo consisten en una manifestación peculiar de la acción del sistema cognitivo humano. Así, al igual que la mente humana, los sistemas documentales no se limitan a identificar y describir la forma y el contenido de los documentos, sino que se dedican sobre todo a organizar el conocimiento que albergan mediante su clasificación y ordenación, desvelando y proponiendo los diversos tipos de relaciones que se producen entre sus contenidos, de acuerdo con unos criterios selectivos y relacionales fijados previamente. Para realizar tal tarea, el gestor de la información perfecciona y controla el sistema usado naturalmente por el hombre, auxiliándose de unos instrumentos conocidos como lenguajes documentales, que contienen las clases autorizadas y establecen los tipos de relaciones autorizadas.

La utilización de criterios selectivos durante la representación y organización documental del conocimiento es ineludible, pero no deja de entrañar una serie de problemas. Por ejemplo, si la selección abstractiva delimita el ámbito en que fijamos la atención hasta el punto que pasamos por alto la mayoría de las propiedades de las cosas ¿cómo podemos saber que no eliminamos parámetros cruciales o decisivos en la representación del contenido de un documento? O durante la construcción de lenguajes documentales, si el documentalista dirige su representación del universo a clasificar por criterios condicionados por sus perspectivas particulares, por las necesidades del servicio en que opera o por los límites del sistema de clasificación empleado ¿no puede suceder que todas esas necesidades, intereses, perspectivas o límites le permitan identificar y considerar sólo una pequeñísima fracción de los hechos involucrados? ¿Hasta qué punto con el uso de criterios selectivos durante la clasificación no actuamos con una estrecha mentalidad provinciana o de campanario?

Los riesgos y los límites del uso de esos criterios selectivos durante la clasificación documental son evidentes. Spinoza decía: «toda determinación es una negación», recuperando la tesis filosófica clásica según la cual el conocimiento es siempre limitación. Sin embargo, en la razón del origen de esos problemas se encuentra el alivio, si somos conscientes de que incluso las formas superiores de conocimiento, como la ciencia, operan siempre dentro de unos límites predefinidos de acción e investigación, trabajando por etapas y disecciones en un avance lento, que no pueden abarcar y comprender todos los elementos de la realidad y

Scire. $1: 1$ (en.-jun. 1995). 
todas sus relaciones como si un ser omnisciente dirigiera sus actos. La toma de conciencia de estos límites debe tranquilizar tanto al científico como al documentalista, porque si es capaz de considerar su existencia en un sentido positivo, puede convertir esos límites y obstáculos en aliados suyos. Si consideramos que el conocimiento es a la vez una búsqueda de la verdad y un intento de alcanzar la completa y verdadera naturaleza de las cosas — si entendemos, por ejemplo, que el proceso documentario intenta captar, recuperar y evitar la pérdida de todas las informaciones contenidas en un documento-, conocer la existencia de que hay límites impoderables, que se avanza rompiendo barreras, que la representación de la realidad es siempre precaria y sometida a verificabilidad, a reforma y profundización, todo ésto nos puede coadyuvar a destruir los obstáculos y avanzar haciendo explícito que la selección de unos parámetros siempre implica la existencia de límites. En consecuencia, nos autoriza a introducir, conscientemente, otros parámetros cuando en un momento posterior consideremos insuficiente nuestro conocimiento de la realidad o del fondo documental; es decir, nos permite reformar de modo controlado nuestra herramienta de clasificación, evolucionando hacia el uso de otras más complejas.

El reconocimiento de la existencia de un componente subjetivo en toda clasificación documental, sea universal o especializada, no debe empujarnos a abandonar el intento de erigir la organización del conocimiento que propone sobre una base racional, abriendo el paso al irracionalismo filosófico, y, por tanto, asumiendo una actitud que puede provocar incluso la negación de la misma posibilidad de clasificar a partir de criterios firmes y controlados, como encontramos de modo implícito en formulaciones como la del filósofo Michel Foucault. La solución consiste en construir clasificaciones documentales cuyas características se correspondan, para ser operativas, con la imagen que una comunidad tiene de la realidad, pero no asumiendo acríticamente la visión que difunden los sistemas de las ciencias y las taxonomías de su época, sino analizando, de acuerdo con el principio del literary warrant, cómo se refleja realmente esa imagen en la literatura sobre la que va a actuar, ya que su función será representar y organizar el conocimiento contenido en los documentos. Evidentemente, se podrá elegir entre diversos criterios para construir las clases de una clasificación, ya que las entidades a clasificar poseen innumerables características, siempre que se discriminen manteniendo una coherencia en su elección y atendiendo a su pertinencia respecto a la relevancia que tienen en la realidad y a los objetivos que persigue el clasificador. El hecho innegable de que cada clasificador puede hacer una clasificación distinta pero igual de correcta y útil de una misma realidad, no debe ser considerado una deficiencia de la actividad clasificadora, sino el testimonio de su capacidad de adaptación a las necesidades que debe servir, si su constructor sabe analizar correctamente el estado del conocimiento en un momento dado, descu-

Scire. $1: 1$ (en.-jun. 1995). 
brir y adaptarse a las necesidades informativas de su entorno y respetar una serie de principios básicos, como la exhaustividad en la división del universo clasificado y el respeto de la relación indirecta entre el crecimiento de la extensión y el incremento de la intensión de las clases según se desciende en la jerarquía.

\section{La clasificación documental como proceso analítico-sintético: la complementariedad de la clasificación y la indización}

No obstante, el concepto de clasificación documental no se elabora, habitualmente, a partir de la observación de las operaciones mentales que se llevan a cabo y los recursos cognitivos que se ponen en acción durante su ejercicio, es decir, a partir del análisis del proceso intelectual que le sirve de base, como proponemos en estas páginas. La mayoría de los autores limitan su conceptualización a la descripción bien de las actividades en las que se manifiesta, bien de las funciones que asume dentro de un sistema documental. Guinchat y Menou ilustran la primera posición cuando definen la clasificación documental como la «operación que describe el contenido de un documento determinando un tema principal y a veces uno o dos temas secundarios que se traducen a unos términos más apropiados que figuran en el lenguaje documental que se utiliza» (Guinchat y Menou, 1981, p. 143). De modo más conciso, Meetham la define como «una brevísima descripción temática del documento» (Meetham, 1975, p. 77. Ref. García Gutiérrez, 1984, p. 165). Por su parte, la bibliografía norteamericana, la inglesa y la hindú inciden en la exposición de su utilidad tanto para facilitar la colocación física de los documentos de un fondo, de acuerdo con el modo de acceso más usual y cómodo para cada tipo de usuario, como para garantizar una recuperación rápida y eficaz de la información que éstos contienen, gracias a la construcción de un fichero con un orden sistemático basado en un esquema de clasificación, que permite establecer una concordancia entre la notación que se asigna a los documentos para su identificación y los términos con que se formula una búsqueda (Berwick Sayers, 1964, 1; Buchanan, 1979, 9-14; Wynar, 1985; Kumar, 1989, p. 1-13). Resumiendo, la clasificación documental se presenta como la disposición de un conjunto de documentos en grupos diversos pero relacionados entre sí, de modo acorde a su contenido temático, a partir de la aplicación de un sistema de clasificación previamente elegido, que consiste en una estructura metódica de clases ligadas entre sí sobre la base de la posesión de una serie de caracteres comunes (Rowley, 1992, p. 176-177). En la misma línea se sitúa Carrión, por citar un autor español, cuando se refiere a la clasificación documental como el proceso de «colocar un libro (un documento, etc.) en una clase dentro de un esquema o bien agrupar los libros por características comunes y siempre por razón de su contenido» (Carrión, 1990, p. 245). Lo cual conduce a identificar la clasificación documental con la organización de la colección de una biblioteca

Scire. $1: 1$ (en.-jun. 1995). 
sobre la base de la representación de la materia o contenido temático más relevante de cada documento, mediante el uso de las convenciones y términos presentes en un lenguaje documental de estructura jerárquica o facetada.

Esta posición reproduce una actitud generalizada a lo largo de la historia de la Documentación, de construcción y modificación de sus conceptos y principios científicos a partir, preferentemente, del análisis de las experiencias y las técnicas de trabajo concretas en los diversos tipos de centros de información, en lugar de adoptar un paradigma que asiente como premisas metodológicas el valor de la comparación y de la dialéctica entre la observación de la realidad y su racionalización en el marco de una teoría previa. En el caso concreto de la Organización del Conocimiento, la mayoría de los autores presentan los conceptos de clasificación e indización ocupándose, exclusivamente, aunque no siempre de modo explícito, de la observación de la actividad de representación del contenido de un documento y el tipo de lenguaje documental más usuales en el centro de información del que se ocupan en sus obras. En concreto, la asignación de materias mediante el empleo de sistemas de clasificación jerárquicos o facetados en las bibliotecas. La identificación del origen o la función de la oficina productora de un documento de archivo mediante el auxilio de un sistema de clasificación que reproduce la estructura orgánica o funcional de la institución generadora del fondo, como resultado de su actividad natural. Y la retención, en los centros de documentación, de los conceptos que estructuran la materia de un documento con la suficiente carga informativa para permitir una recuperación posterior pertinente, mediante el empleo de un lenguaje de estructura combinatoria compuesto por unidades conceptuales cargadas de pleno sentido.

Así, la clasificación de los documentos ocupa, junto con la catalogación, un lugar central en la descripción del proceso documental que se efectua en las bibliotecas y los archivos. En cambio, en la exposición de las operaciones de la cadena documental planteada desde el análisis de las tareas que se llevan a cabo en un centro de documentación, se suele incidir en la presentación de la indización del contenido de los documentos como la tarea fundamental. Lo cual provoca dos fenómenos no deseables. Por un lado, la identificación de la clasificación con el carácter peculiar que adopta en una biblioteca o un archivo, sin considerar la posibilidad de que la clasificación bibliográfica y la archivística constituyan otras tantas especies de un mismo género: la clasificación documental. Y, por otro, la incidencia en sus diferencias con la indización, en lugar de atender a sus semejanzas; obstaculizando, de este modo, una comprensión de la clasificación documental integradora de todas las operaciones documentales en las que se manifiesta y con las que se relaciona, así como válida para cualquier centro de información.

Sin embargo, si se atiende al carácter de los procesos intelectuales que fun-

Scire. 1 : 1 (en.-jun. 1995). 
damentan las tareas de indización y clasificación, no sólo observamos que sus diferencias son adjetivas, sino que se tratan de una actividades complementarias que aparecen en todo proceso de representación y organización del contenido de un documento, con independencia del lenguaje documental empleado. La confusión de la clasificación y la indización con el uso de uno u otro tipo de lenguaje documental, se debe al olvido de que, de modo previo al empleo de esas herramientas, existe un proceso de identificación y representación mental del contenido de los documentos, que combina ambas técnicas. Como apunta Langridge, no debemos confundir el empleo del término clasificación como un proceso fundamental de pensar y operar, con la descripción del modo de uso de un sistema destinado a incluir la colocación de documentos en un orden clasificado (Langridge, 1992, 63 y 71).

Evidentemente, el clasificador se limita a representar el contenido de un documento del modo más general posible, caracterizando el tema y la forma de conocimiento dominante mediante la extracción, expresión codificada e inclusión en una jerarquía de las materias presentes, conforme a las convenciones de un sistema de clasificación. En cambio, el indizador se dedica a describir con detalle el contenido del documento mediante la selección de los conceptos con mayor carga informativa y más pertinentes respecto al texto y las necesidades del servicio de información en el que se encuentra, para su representación con unos términos de indización extraídos, generalmente, de un lenguaje combinatorio. Es decir, la clasificación se sitúa en el nivel más sintético posible, reduciendo a una unidad las diversas partes componentes de un documento, mientras que la indización adopta una perspectiva analítica o de disección frente al documento.

Pero de estas diferencias no se debe deducir que ambas operaciones documentales son irreconciliables. Por el contrario, su complementariedad se afirma si atendemos a cómo se integran las facultades analíticas y sintéticas en el intelecto humano durante el proceso de generación de conceptos. En primer lugar, el sujeto, gracias a su capacidad abstractiva, emprende un proceso de análisis, consistente en la búsqueda y objetivación de los elementos y relaciones que componen la estructura de un todo observado (fenómeno, experiencia, texto, etc.) mediante el desarrollo, por este orden, de tres actividades: identificación y enumeración de los elementos y las partes de un objeto, distinguiendo los sustanciales de los fútiles; hallazgo de los diversos tipos de conexiones (inclusión, oposición, causa-efecto, correspondencia, discrepancia, etc.) entre los elementos identificados como relevantes; y descubrimiento de los principios que rigen la organización del fenómeno o información que se observa. Para, en un segundo momento, mediante la deducción de relaciones abstractas entre los objetos homogénos a partir de los elementos obtenidos durante el análisis de cada uno de ellos, proceder, gracias el auxilio de las capacidades de comparación y generali-

Scire. $1: 1$ (en.-jun. 1995). 
zación, a la reducción o síntesis de esos objetos a una unidad, hasta obtener una nueva estructura lógica que no existía previamente.

En consecuencia, y por analogía, del mismo modo que el hombre sostiene la actividad sintética que permite la construcción de conceptos, sobre una actividad analítica previa de la realidad observada, el documentalista mejorará los resultados de su labor de indización y clasificación, si sabe conjugar el carácter analítico de la primera con el sintético de la segunda - lo cual ya hace de hecho de modo inconsciente, y, por tanto, sin obtener los beneficios que resultan de su control-, ya que se basan en unas actividades mentales no excluyentes, sino complementarias.

La indización no es tanto una alternativa a la clasificación como un acción ineludible para efectuar ésta, ya que la descomposición que el clasificador emprende, inconscientemente, de una materia en sus elementos más simples, permite precisar su naturaleza y convertir su representación en un momento de reunificación e integración. La indización es una actividad consustancial al ser humano, en cuanto que éste escruta continuamente su entorno para extraer indicios que le permitan conocer, adaptarse y actuar. Si bien, como apunta García Gutiérrez, se trata de una operación sin utilidad para la representación y organización documental, si su realización no se sistematiza y sujeta a una norma (García Gutiérrez, 1989, p. 375). Por consiguiente, es deseable su plena objetivación durante el proceso documental, aun cuando la finalidad sea la clasificación, $\mathrm{y}$, aun a sabiendas, de que no todo fondo clasificado será por ello un fondo indizado con idéntica profundidad, ya que el grado de ésta depende del tiempo y la intensidad dedicados por el documentalista y del carácter y la riqueza léxica del lenguaje documental empleado. Pero no sólo la indización es útil para lograr una correcta clasificación, sino que la clasificación, por tratase de un proceso sintético, se convierte en un rellano o momento de cierre y de reconsideración en el proceso analítico de indizar, que, precisamente por esa elevación sobre los conceptos descubriendo su integración en un mismo o cercanos campos semánticos, permite guiar mejor la indización. Indización y clasificación se complementan del mismo modo que el análisis conduce a la síntesis y la síntesis perfecciona el análisis, conformando un ciclo en el que es absurdo oponer mutuamente ambas actividades.

Ranganathan, debido a que se centró en el estudio de los fundamentos intelectuales de la organización documental, fue pionero en la construcción de una concepción del proceso de clasificación integradora de las operaciones de análisis y síntesis, como se vislumbra en su distinción y presentación de tres estadios en el proceso de clasificación documental. En primer lugar, la fase eidética (idea plane), consistente en el análisis de la materia principal de un documento mediante el descubrimiento de la forma de conocimiento dominante, las catego-

Scire. 1 : 1 (en.-jun. 1995). 
rías usadas, los conceptos que las expresan y las relaciones establecidas por el autor; así como la discriminación de posibles formas de conocimiento y temas secundarios, siguiendo un mismo método de descomposición. En segundo lugar, la fase verbal (verbal plane), que se caracteriza por el examen de un plan de clasificación para proceder a la recomposición y verbalización de la representación intelectual del contenido mediante los conceptos autorizados en el sistema documental en el que nos encontramos. Y, en tercer lugar, la fase notacional (notational plane), destinada a asignar y construir una notación o una cadena de términos de indización en lenguaje natural, de acuerdo con las reglas del esquema del lenguaje documental empleado (Ranganathan, 1957).

Esta presentación de la actividad clasificatoria se funda en una crítica previa de la forma de representar el conocimiento consistente en la identificación del tema de un documento con una materia unidimensional extraída de un sistema de clasificación jerárquico. Para el bibliotecario hindú el tema de un documento tiene una naturaleza compuesta, ya que se trata de un agregado de materias específicas, donde cada cada una de las cuales es el tema principal tratado desde una perspectiva particular y formado por una composición más o menos compleja de conceptos simples. Pero la complejidad de estos conceptos no se limita a una diversidad y riqueza de orden físico-estructural, sino que también se basa en la existencia de una riqueza de relaciones entre ellos. Es decir, un tema no incluye sólo una multiplicidad de referentes físico-estructurales de las entidades clasificadas, con su correlato en la realidad, sino que por ser producto de una reflexión humana, cada concepto y el conjunto de todos ellos remiten también a una serie diversa de elementos tales como características, funciones procesos, operaciones, propiedades... introducidos por el autor del documento y que, por consiguiente, se deben desvelar durante la indización. Estos elementos se conocen como facetas de la materia, basándose su existencia en el hecho de que el contenido de un concepto no se puede comprender de modo aislado del resto, sino que la plena comprensión de su significado sólo es posible a partir de su agrupación con otros vecinos en un mismo campo semántico y el desvelamiento de las relaciones que teje con éstos, así como las que establece el campo al que pertenece con la totalidad de los campos del universo (Vickery, 1975, p. 8-10).

\section{Conclusión}

En resumen, por una parte, al clasificador corresponde decidir y representar el contenido de un documento antes de traducirlo a las convenciones de un sistema de organización particular; y, por otra, esa representación se consigue mediante un proceso donde se complementan las actividades analíticas y sintéticas, destinado a diseccionar y reconstituir ese contenido de modo acorde con su naturaleza compuesta y relacional, trasunto del carácter propio de los fenómenos

Scire. 1 : 1 (en.-jun. 1995). 
reales. Al igual que el recurso cognitivo de clasificar, la clasificación documental aparece, por tanto, como un proceso analítico-sintético de discriminación y agrupación en clases de la representación conceptual del contenido informativo de los documentos de un fondo, que resulta del ejercicio de dos operaciones complementarias, denominadas, comúnmente, indización y clasificación en sentido estricto, que se integra en el marco de un proceso superior destinado a permitir la conservación y difusión del saber humano, denominado organización del conocimiento.

El clasificador puede elegir como criterios de selección y relación de las clases, bien el modo de generación de los documentos, la forma de conocimiento dominante, las materias que los caracterizan y los conceptos que constituyen a éstas atendiendo a diversas perspectivas o facetas, o bien la suma de algunos o de todos ellos. Como la función de la clasificación es lograr una representación y organización del contenido de los documentos que permita la posterior recuperación de los más adecuados para responder a la consulta del usuario de un servicio de información sobre un tema determinado, la selección de los términos de clasificación se efectua teniendo en cuenta dos perspectivas. En primer lugar, su adecuación al contenido informativo del documento, por lo que esos términos deben ser exhaustivos, profundos, pertinentes, precisos y coherentes. Y, en segundo lugar, las necesidades informativas que condicionan la formación del fondo documental, de modo que su previa identificación condiciona la elección de los principios que ha de regir la clasificación y el diseño de las facetas mediante las que se interroga al documento para proceder al descubrimiento de su contenido.

Las unidades de representación del contenido se expresan mediante unos términos formalizados en lenguaje natural o codificado, extraídos de un peculiar instrumento lógico-lingüístico: un lenguaje documental, cuyo tipo concreto ha sido previamente elegido entre una variada gama generada por la diversidad en su estructura y en el grado de control de su vocabulario, destinado a permitir la traducción de las preguntas formuladas a un sistema documental durante el proceso de recuperación a las convenciones utilizadas durante el tratamiento. Estas herramientas auxiliares de la clasificación documental se denominan, genéricamente, lenguajes, por tratarse de una colección de términos y símbolos con una morfología normalizada, que remiten a conceptos que se relacionan entre sí tras proceder a un control más o menos rígido de su significado, y que se estructuran y combinan mediante un conjunto de reglas lógicas predefinidas del que resulta una sintaxis más o menos compleja. Recibiendo el apelativo de documentales tanto por su función auxiliar de la representación y organización del contenido de los documentos en un sistema de información, como por generarse principalmente a partir del tratamiento de esos documentos. Los lenguajes documentales se caracterizan, generalmente, por una simplicidad de organización y una econo-

Scire. $1: 1$ (en.-jun. 1995). 
mía de signos basadas en el principio de la univocidad entre el objeto real, su representación conceptual y su expresión lingüística, con objeto de conseguir un proceso de tratamiento documental entrópico, es decir, donde cada signo aporta la mayor cantidad posible de información, al tiempo que evita la redundancia no suministrando la información ya transmitida por otros elementos.

En definitiva, proponemos un acercamiento al concepto de clasificación documental que no se limite ni a la descripción de sus funciones y resultados, ni a su confusión con una presentación de las peculiaridades de los diversos tipos de lenguajes documentales, ni a la exposición del modo de uso de los sistemas de clasificación bibliográficos y archivísticos. Frente a estas perspectivas descriptivistas y reduccionistas, planteamos la necesidad urgente de pensar y construir un concepto de clasificación documental más ambicioso e integrador, que atienda a las dos dimensiones implícitas en su naturaleza: la operativa, es decir, el proceso intelectual que se produce para llevarla a cabo; y la instrumental, es decir, la herramienta de estructura lógica y lingüística que se emplea como auxiliar. De modo que el edificio conceptual resultante se articule, como mímino, en torno a estos ejes: el estudio de las habilidades intelectuales desplegadas durante su ejercicio, que servirá de cimiento; el análisis de las experiencias y peculiaridades de la clasificación en los distintos sistemas y centros de información, que aportará los materiales constructivos; y la investigación de los principios científicos de las técnicas de la representación y organización del conocimiento, que actuará como argamasa. Sólo ésto facilitará el diseño de una estrategia del proceso de clasificación documental capaz de permitir la creación de lenguajes documentales eficaces, la adquisición de criterios para decidir la superioridad de un tipo de lenguaje sobre otro en los diferentes contextos, el trasvase eficaz de habilidades e instrumentos de representación y organización documental entre los diversos centros, la mejora de los productos documentales y la optimización de los recursos humanos en los sistemas de información durante las tareas del análisis de contenido, gracias a la selección y combinación de las tácticas más adecuadas para cada situación documental.

\section{Referencias}

Barreau, H. (1990). L’Épistemologie. París : Presses Universitaires de France, 1990.

Berwick Sayers, W. C. (1964). Manual of classification for librarians and bibliographers. 3rd. ed. New York : Andre Deutsch, 1964.

Blanché, R. (1973). La epistemología. Barcelona : Oikos-tau, 1973.

Bunge, M. (1983). La investigación científica. 2a . ed. Barcelona : Ariel, 1983.

Carrión Gútiez, M. (1990). Manual de bibliotecas. Salamanca, Madrid : Fundación Germán Sánchez Ruipérez, 1990.

Dahlberg, I. ( 1991). Philosophical foundations of conceptual ordering systems. //

Scire. $1: 1$ (en.-jun. 1995). 
Documentary languages and databases. Frankfurt/Main : Indeks Verlag, 1991. 102119.

Dahlberg, I. (1992). Knowledge organization and terminology : Philosophical and linguistic bases. // International Classification. $19: 2$ (1992) 65-71.

Dewey, J. (1989). Cómo pensamos : Nueva exposición de la relación entre pensamiento reflexivo y proceso educativo. Barcelona : Paidós, 1989.

Ferrater Mora, J. (1982). Diccionario de Filosofía. Madrid : Alianza editorial, 1982. 4 vols.

García Gutiérrez, A. (1984). Lingüística documental : Aplicación a la documentación de la comunicación social. Barcelona : Mitre, 1984.

García Gutiérrez, A. (1989). Nuevos parámetros para una teoría de la indización de documentos. // López Yepes, J. (comp.). Fundamentos de información y documentación. Madrid : EUDEMA, 1989. 375-384.

García Gutiérrez, A. (1990). Estructura lingüística de la documentación: teoría y método. Murcia : Universidad de Murcia, Secretariado de Publicaciones, 1990.

García Marco, F. J. ; Esteban Navarro, M. A. (1993). On some contributions of the Cognitive Sciences and Epistemology to a Theory of Classification. // Knowledge Organization. $20: 3$ (1993) 126-132.

Gardner, H. (1988). La nueva ciencia de la mente. Historia de la revolución cognitiva. Barcelona : Paidós, 1988.

Guinchat, C. ; Menou, M. (1981). Introduction générales aux sciences et techniques de l'information et dela documentation. Paris : Les Presses del'Unesco, 1981.

Hempel, C. G. (1988). La explicación científica. Estudios sobre la filosofía de la ciencia. Barcelona : Paidós, 1988.

Kumar, K. (1989). Theory of classification. 4th ed. rev. New Delhi : Vikas, 1989.

Langridge, D. W. (1989). Subject analysis : Principles and procedures. Londres : BowkerSaur, 1989.

Landgridge, D. W. (1992). Classification : its kinds, systems, elements and applications. Londres : Saur, 1992.

López-Huertas Pérez, M. ${ }^{a}$ J. (1991). Lenguajes documentales : terminología para un concepto". // Boletín de la Anabad. 41 : 2 (1991) 171-188.

Maniez, J. (1987). Les langages documentaires et classificatoires : conception, construction et utilisation dans les systèmes documentaires. París : Les Éditions d'Organisation, 1987.

Meetham, Roger (1975). Recuperación de información. Buenos Aires : Ateneo, 1975.

Monserrat, J. (1984). Epistemología evolutiva y teoría de la ciencia. Madrid : UPCM, 1984.

Mosterin, J. (1984a). La estructura de los conceptos científicos. // Conceptos y teorías en la ciencia. Madrid : Alianza Editorial, 1984. 11-39.

Mosterin, J. (1984b) Taxonomía formal. // Conceptos y teorías en la ciencia. Madrid : Alianza Editorial, 1984. 41-64.

Ranganathan, S. R. (1957). Prolegomena to library classification. 2nd. ed. London : The

Scire. 1 : 1 (en.-jun. 1995). 
Library Asssociation, 1957.

Ranganathan, S. R. (1989). Philosophy of Library Classification. Bangalore : Sarada Ranganathan Endowment for Library Science, 1989.

Rowley, J. E. (1992). Organizing Knowledge: an introduction to information retrieval. Aldershot : Ashgate, 1992 ( $2^{\text {a }}$ ed. rev.)

Runes, D. (1985). Diccionario de Filosofía. Barcelona : Ariel, 1985.

Vickery, B. C. (1975). Classification and indexing in science. 3rd ed. Londres : Butterworths Scientific Publications, 1975.

Wartorfsky, M. W. (1973). Introducción a la filosofía de la ciencia. Madrid : Alianza Editorial, 1973.

Wynar, B. S. (1985). Introduction to cataloguing and classification. 7th ed. Littleton : Libraries Unlimited, 1985.

Scire. 1 : 1 (en.-jun. 1995). 